\title{
OVERRINGS OF COMMUTATIVE RINGS. II. INTEGRALLY CLOSED OVERRINGS( $\left.{ }^{(}\right)$
}

\author{
BY \\ EDWARD D. DAVIS
}

Introduction. All rings in this paper are commutative with identity element, and all subrings possess the identity element of the containing ring. An overring of an integral domain denotes here a subring of the quotient field containing the given ring. We assume the reader conversant with the elements of commutative (Noetherian) ring theory presented in the textbook of Zariski and Samuel [8], especially in those passages dealing with quotient rings, integral extensions, Dedekind rings and the elementary theorems on valuation rings. Let that book serve as a general reference for the "well-known" definitions and results we introduce here with little or no comment.

It is a fact that every overring of a Prüfer ring (in the Noetherian case, Dedekind ring) is also a Prüfer ring, and, a fortiori, integrally closed. Further, for some Prüfer rings (e.g., the ring of integers) all overrings are rings of quotients. Our purpose here is the study of rings with such properties, namely: P-domains, integral domains for which all overrings are integrally closed, and $Q$-domains, integral domains for which all overrings are rings of quotients. In $\S 1$ we see that $P$-domains are indeed Prüfer rings and that $Q$-domains are a special class of Prüfer rings (in the Noetherian case, exactly those Dedekind rings with torsion class group). In $\$ 2$ and $\S 3$ we consider " $P$-rings" and " $Q$-rings" in the context of rings with divisors of zero. $\S 3$, which deals exclusively with Noetherian rings, is essentially a consideration of the notion "Dedekind rings" in the presence of zero-divisors. $\$ 2$ treats a special case of (sometimes) non-Noetherian rings.

Since the results of $\S 2$ and $\S 3$ include as special cases many of the results of $\S 1$, the reader is due an explanation of this uneconomical style of exposition. The excuse is twofold: (1) The consideration of integral domains contains the basic ideas of this study and serves to motivate the more general considerations. (2) The essentially simple ideas involved here tend to become obscured by the technicalities required in the presence of zero-divisors. We hope that this choice of exposition will produce a gain in clarity sufficient to compensate for the increased length. We shall attempt to avoid duplication of arguments by only

Received by the editors June 25, 1962.

(1) Portions of this paper appear in the author's doctoral dissertation (University of Chicago, 1961). At Chicago, the author was partially supported by the Office of Ordnance Research; at Yale, by NSF Grant 18916. 
sketching certain generalizations of results already established for integral domains, at least wherever doing so does not seem to result in an outrageous offense against clarity.

I. Integral domains. Recall that a valuation ring is an integral domain with the property: of any two elements, one divides the other. Of the properties of valuation rings, we require for the moment only the following three simple consequences of the definition. (1) The ideals of a valuation ring are linearly ordered by inclusion. (2) If $T$ is an overring of the valuation ring $R$, then $T$ is a valuation ring; if $M$ is the unique maximal ideal of $T$, then $T=R_{M \cap R}$. (3) Valuation rings are integrally closed. A discrete valuation ring is a Noetherian valuation ring; that is, a principal ideal domain with exactly one prime. An integral domain $R$ is a Prüfer ring if for each maximal ideal $M, R_{M}$ is a valuation ring. A Dedekind ring is a Noetherian Prüfer ring; that is, a Noetherian integral domain such that the local ring corresponding to each of its maximal ideals is a discrete valuation ring. Arbitrary Prüfer rings are perhaps not such well-known objects; but the only Prüfer ring properties that we shall require follow easily from the properties of valuation rings. One such is as follows: If $P$ is a prime of the Prüfer ring $R$, then the primes contained in $P$ are linearly ordered by inclusion. This fact follows from property (1) of the valuation ring $R_{P}$ and the one-to-one correspondence between the prime ideals of $R_{P}$ and those primes of $R$ contained in $P$.

Definition. Let $\Delta$ be a set of prime ideals of the integral domain $R$. $R_{\Delta}=\bigcap\left\{R_{P} \mid P \in \Delta\right\}$. (It is well known that $R=R_{\Delta}$, where $\Delta$ is the set of (maximal) prime ideals of $R$.)

LEMMA 1. If the integral closure of the integral domain $R$ is a ring of quotients of $R$, then $R$ is already integrally closed.

Proof. Let the integral closure of $R$ be $R_{S}$, where $S$ is a multiplicatively closed subset of nonzero elements of $R$. For $s \in S, 1 / s$ is integral over $R$. Hence:

$$
(1 / s)^{t}=r_{1}(1 / s)^{t-1}+r_{2}(1 / s)^{t-2}+\cdots+r_{t}, \quad r_{i} \in R .
$$

Multiplication of this equation by $s^{t-1}$ shows that $1 / s \in R$. That is, the elements of $S$ are units in $R$, and $R=R_{S}$. Lemma 1 is now established.

The observation that a ring of quotients of an integrally closed integral domain is also integrally closed combines with Lemma 1 to prove:

Proposition 1. Q-domains are P-domains.

PROPOSITION 2. The following statements are valid for the Prüfer ring $R$. (1) Every overring of $R$ is a Prïfer ring. (2) Every overring of $R$ is of the form $R_{\Delta} ; \Delta$ may be taken to be a set of pairwise co-maximal primes of $R$. (3) If $R$ has only finitely many maximal ideals, then it is a Q-domain. 
Proof. Let $T$ be an overring of $R$. For each maximal ideal $M$ of $T$, $T_{M}$ is an overring of the valuation ring $R_{M \cap R}$; hence $T_{M}$ is a valuation ring. Thus $T$ is a Prüfer ring, and (1) is established. Observe that since $\left(M T_{M}\right) \cap R_{M \cap R}=(M \cap R) R_{M \cap R}, T_{M}=R_{M \cap R}$. Now $T=\bigcap\left\{T_{M} \mid M\right.$ maximal ideal of $T\}$. Then $T=R_{\Delta}$, where $\Delta=\{M \cap R \mid M$ maximal ideal of $T\}$. If $M$ and $N$ are distinct maximal ideals of $T$, then there is no containment relation between $T_{M}$ and $T_{N}$; and therefore none between $R_{M \cap R}$ and $R_{N \cap R}$; and therefore none between $M \cap R$ and $N \cap R$. That is, the members of $\Delta$ are pairwise comaximal. This observation completes the proof of (2). If $R$ has but finitely many maximal ideals, then the set $\Delta$ must be finite. In this case $R_{\Delta}=R_{S}$, where $S$ is the complement in $R$ of the set-theoretic union of the members of $\Delta$. (3) is now proved.

REMARKS. 1. In the Dedekind ring case every such $T$ is in fact a Dedekind ring; an elementary proof of this fact appears in [3]. 2. Furthermore, in the Dedekind ring case $\Delta$ is unique: distinct sets $\Delta$ give distinct overrings. (This fact will appear implicitly in the proof of Theorem 2.) 3. In a special case, which includes Noetherian rings, the converse of the second assertion of Proposition 2 is valid (Corollary 2 of Theorem 1). This fact adds yet another entry to the long list of characterizations of Dedekind rings.

Theorem 1. An integral domain is a Prüfer ring if, and only if, all of its overrings are integrally closed. (And consequently: a Noetherian integral domain is a Dedekind ring if and only if all of its overrings are integrally closed.)

Proof. We have seen in Proposition 2 that every overring of a Prüfer ring is an intersection of integrally closed rings (actually valuation rings) and is therefore integrally closed. Conversely, suppose that $R$ is a $P$-domain. We must show that $R_{M}$ is a valuation ring for each maximal ideal $M$; that is, for each (nonzero) pair $x, y$ in $R_{M}$, we must show that $x / y \in R_{M}$ or $y / x \in R_{M}$. Now $R_{M}$ clearly inherits the $P$-domain property. Therefore, since $R_{M}\left[x^{2} / y^{2}\right]$ is integrally closed, and $x / y$ is integral over $R_{M}\left[x^{2} / y^{2}\right]$ we obtain:

$$
x / y=r+r_{1}\left(x^{2} / y^{2}\right)+\cdots+r_{t}\left(x^{2} / y^{2}\right)^{t}, \quad r, r_{i} \in R_{M} .
$$

Multiplication of this equation by $(y / x)(r y / x)^{2 t-1}$ yields an equation of integral dependence for $r y / x$ over $R_{M}$. Hence $y r / x \in R_{M}$. If $y r / x$ is a unit in $R_{M}$, then $x / y \in R_{M}$. Otherwise $y r / x \in M R_{M}$; in this case multiply $\left(^{*}\right)$ by $(y / x)^{2 t}$ to obtain

$$
(1-y r / x)(y / x)^{2 t-1}=r_{1}(y / x)^{2 t-2}+r_{2}(y / x)^{2 t-4}+\cdots+r_{t} .
$$

Since $(1-y r / x)$ is a unit in $R_{M}$, this is an equation of integral dependence for $y / x$ over $R_{M}$. Hence $y / x \in R_{M}$. This concludes the proof of Theorem 1 .

Lest the reader think the hypothesis of Corollary 1 artificially contrived, we note in advance that 1-dimensional Noetherian domains do satisfy this condition 
[6]; the second assertion of the corollary is the converse of this theorem. In $\S 2$ we shall record generalizations of both the theorem and its converse for rings with divisors of zero.

COROLlaRY 1. Let $R$ be an integral domain with property that each of its overrings is of the form $T_{\Delta}$, where $T$ is an integral extension of $R$. Then the integral closure of $R$ is a Prüfer ring. Further, if $R$ is Noetherian, then it is of dimension at most 1 .

Proof. Suppose that $W$ is an overring of $\bar{R}$, the integral closure of $R$. By the hypothesis, $W=T_{\Delta}$, where $T$ is an overring of $R$ contained in $\bar{R}$. Note that for $P \in \Delta, T_{P}$ contains $\bar{R}$ since $T_{P}$ contains $W$; let $M=P T_{P} \cap \bar{R}$. Then $T_{P} \supset \bar{R}_{M} \supset T_{P}$; that is $T_{P}=\bar{R}_{M}$, an integrally closed ring. Therefore $W$ is integrally closed (since it is an intersection of integrally closed rings). It now follows from Theorem 1 that $\bar{R}$ is a Prüfer ring. Suppose further that $R$ is Noetherian. If $N$ is a prime ideal in $R$ of rank greater than 1 , then $N$ contains infinitely many rank 1 primes $\left\{P_{i}\right\}$. For each of the chains $N \supset P_{i}$ there is a chain of primes $\bar{N}_{i} \supset \bar{P}_{i}$ in $\bar{R}$ such that $\bar{N}_{i} \cap R=N$ and $\bar{P}_{i} \cap R=P_{i}$. The set $\left\{\bar{N}_{i}\right\}$ is infinite: If $\bar{N}_{i} \supset \bar{P}_{j}$ for $i \neq j$, then since $\bar{R}$ is a Prüfer ring, there is a containment relation between $\bar{P}_{i}$ and $\bar{P}_{j}$. But a theorem of Chevalley [1] asserts: For any Noetherian integral domain $R$, there are only finitely many primes of $\bar{R}$ lying over each prime of $R$. The contradiction of Chevalley's theorem then proves that $R$ has no prime of rank greater than 1.

COROLlary 2. Let $R$ be an integral domain in which every prime ideal has finite rank. Then $R$ is a Prüfer ring if all of its overrings are of the form $R_{\Delta}$. Consequently, a Noetherian integral domain with this condition on its overrings is a Dedekind ring.

Proof. By the first assertion of Corollary 1, the integral closure of $R$ is a Prüfer ring; it remains to show that $R$ is integrally closed. Proceeding by induction on rank $M$, we shall show that $R_{M}$ is integrally closed for every prime ideal $M$. Let $T$ be the integral closure of $R_{M}$, and suppose that $R_{M} \neq T$. Now $T=R_{\Delta}$, where $\Delta$ is a subset of the primes properly contained in $M$; for if $P \notin M$, then $R_{M} \notin R_{P}$, and if $M \in \Delta$, then $R_{M}=T$. By the induction hypothesis, $R_{P}$ is integrally closed for each $P$ properly contained in $M$. Hence: $T \subset \bigcap\left\{R_{P} \mid P\right.$ properly contained in $\left.M\right\}$ $\subset R_{\Delta}=T$. It follows that $\Delta$ may be taken to be the set of primes properly contained in $M$. Now any proper overring of $R_{M}$ is of the form $R_{\Delta^{\prime}}$, where $\Delta^{\prime}$ is a subset of $\Delta$. Since $R_{\Delta} \subset R_{\Delta^{\prime}}$, it is clear that there are no rings properly between $R_{M}$ and $T$. Select $x \in T-R_{M}$; that $T=R_{M}[x]$ is clear. Since $x$ is integral over $R_{M}, T$ is a finitely generated $R_{M}$-module. It follows that $T$ has only a finite number of maximal ideals. (Each maximal ideal of $T$ contains $M T$, and $T / M T$ is a finite-dimensional algebra over $R_{M} / M R_{M}$.) Since each maximal ideal of $T$ of is rank no greater than rank $M$, and $T$ is a Prüfer ring ( $T$ is an overring of a 
Prüfer ring, namely, the integral closure of $R$ ), we can conclude that $T$ contains only a finite number of prime ideals. (We have used here the fact noted earlier that the prime ideals of a Prüfer ring contained in a given prime are linearly ordered by inclusion.) Since every prime ideal of $R_{M}$ is the restriction to $R_{M}$ of a prime ideal of $T$, it follows that the set $\Delta$ is finite. Hence $T=R_{S}$, where $S$ is the complement in $R$ of the set-theoretic union of the members of $\Delta$. Then, a fortiori, $T=\left(R_{M}\right)_{S}$. Hence, by Lemma $1, R_{M}=T$. This contradiction completes the induction step and thereby establishes Corollary 2.

REMARK. The reader has doubtless noticed that the Noetherian version of Corollary 2 is readily established by an appeal to Corollary 1 (to show that $R$ is 1-dimensional) followed by the then trivial observation that each $R_{M}$ must be integrally closed. This line of argument has buried in it Chevalley's theorem which lies much deeper than the arguments given above; the proof of that theorem requires the theory of completions of local rings. The proof given for Corollary 2 has the advantage of being both more general and more elementary than the one just sketched. The author is ignorant of whether this result is valid without the finiteness hypothesis; perhaps some skillful manipulator of transfinite methods can settle this point.

For the remainder of this section we consider a special class of Prüfer rings (Proposition 1 and Theorem 1): $Q$-domains. Let $R$ be an integral domain with quotient field $F$. Recall that a fractional ideal of $R$ is a nonzero, finitely generated $R$-submodule of $F$. The set of fractional ideals under multiplication form a semigroup with identity $(R)$. If $R$ is a Dedekind ring, then this semigroup is a group, and the class group is the group of fractional ideals modulo the subgroup of principal fractional ideals. The following statements are equivalent for a Dedekind ring: 1 . The class group is a torsion group. 2. Some power of each ideal is principal. 3. Every nonzero prime ideal contains an element which lies in no other prime ideal. Furthermore:

Theorem 2. A Noetherian integral domain is a Q-domain if, and only if, it is a Dedekind ring with torsion class group.

Proof. Let $R$ be the ring in question. Suppose first that $R$ is a Dedekind ring with torsion class group. A typical overring of $R$ is of the form $R_{\Delta}$. Let $S$ be the complement in $R$ of the set-theoretic union of the members of $\Delta$. We shall show that $R_{\Delta}=R_{S}$. Now $R_{S}=R_{\Delta^{\prime}}$, where $\Delta^{\prime}$ consists of those primes of $R$ which do not meet $S$. Suppose $P \notin \Delta$. Select an element of $P$ which lies in no other prime ideal; this element must then lie in $S$. Hence $P \notin \Delta^{\prime}$. This shows that $\Delta$ contains $\Delta^{\prime}$. Since $\Delta^{\prime}$ clearly contains $\Delta$, we have that $\Delta=\Delta^{\prime}$, and that consequently $R_{\Delta}=R_{S} . R$ is therefore a $Q$-ring. On the other hand suppose that the class group of $R$ is not torsion. Then $R$ contains a set of primes $\Delta$ and a nonzero prime $M$ such that $M \notin \Delta$ and $M$ is contained in the set-theoretic union of the 
members of $\Delta$. We shall demonstrate that $R_{\Delta}$ cannot be a ring of quotients of $R$. Select $x$, a nonzero element of $M$. If $Q_{1}, \cdots, Q_{t}$ are the members of $\Delta$ which contain $x$, select $y$, an element in $\left(\bigcap\left\{Q_{i}\right\}\right)-M$. (This is possible because $M$ contains no $Q$.) Now for each such $Q$, since $x$ and $y$ lie in the maximal ideal of the 1-dimensional local ring $R_{Q}$, we have that a sufficiently high power of $y$ lies in $x R_{Q}$. Therefore for sufficiently large $n, y^{n} / x$ lies in each $R_{Q}$. Since $x \notin P$ for $P \in \Delta$ and $P$ not a $Q$, we have that $y^{n} / x \in R_{\Delta}$. Now suppose that $R_{\Delta}=R_{S}$. Since for $P \in \Delta, R_{P}$ contains $R_{S}, P$ does not meet $S$; it follows that $M$ does not meet $S$. Since $y^{n} / x \in R_{\Delta}=R_{S}, y^{n} / x=r / s$, where $r \in R$ and $s \in S$. Hence $s y^{n} \in M$-an absurdity, since $s \notin M$ and $y \notin M$. This contradiction shows that $R_{\Delta}$ is not a ring of quotients, and thereby completes the proof of Theorem 2.

Proposition 3 is a technical result which will provide us with several examples of $Q$-domains. In its statement, and in the discussion leading to the corollaries that follow it, we maintain the following special notation: $D$ is an integrally closed integral domain with quotient field $F ; L$ is an algebraic extension field of $F$; $E$ is the integral closure of $D$ in $L$. Observe that for any subfield $H$ of $L$ containing $F, E \cap H$ is the integral closure of $D$ in $H$; if $T$ is an integrally closed subring of $L$, containing $D$, with quotient field $H$, then the integral closure of $D$ in $T$ is the integral closure of $D$ in $H$.

Proposition 3. Suppose that for every subfield $G$ of $L$ finite over $F, E \cap G$ is a $Q$-domain. Then: (1) $E$ is a $Q$-domain. (2) For every subfield $H$ of $L$ containing $F, E \cap H$ is a $Q$-domain. (3) If $T$ is an integrally closed subring of L containing $D$, then $T$ is a ring of quotients of its subring of elements integral over $D$. (4) Every integrally closed subring of L containing $D$ is a $Q$-domain.

Proof. Suppose that $W$ is an overring of $E$. We shall show that $W=E_{S}$, where $S=\{s \in E \mid 1 / s \in W\}$. That $W$ contains $E_{S}$ is clear. Suppose $x \in W$. Let $G=F(x)$. By the hypothesis, $E \cap G$ is a $Q$-domain; $W \cap G$ is therefore a ring of quotients of $E \cap G$. In particular then, $x=e / s$, with $e \in E$ and $s \in S$. So $W=E_{S}$, and (1) is established. To prove (2) we apply (1) with $H$ playing the role of $L$ and $E \cap H$ playing the role of $E$. To prove (3) we observe that $T \cap E$ is the integral closure of $D$ in the quotient field of $T$; by (2) then, $T$ is a ring of quotients of $E \cap T$. This last argument also proves (4) by showing that every integrally closed subring of $L$ containing $D$ is an overring of a $Q$-domain, and is therefore a $Q$-domain.

Now it is a well-known result in number theory that if $D$ is either the ring of integers or $k[x]$, where $k$ is a finite field and $x$ is an indeterminate over $k$, then the integral closure of $D$ in any finite algebraic extension field of $F$ is a Dedekind ring with finite class group. Such integral extensions are then $Q$-domains (Theorem 2). These facts and an application of Proposition 3 yield the following two corollaries. 
COROLlARY 1. Every integrally closed subring of the field of all algebraic numbers is a quotient ring of its subring of algebraic integers. Every integrally closed subring of the field of all algebraic numbers is a Q-domain.

COROLlaRY 2. Let $K$ be a field of nonzero characteristic and of transcendence degree 1 over its prime field $k$. Then every integrally closed subring $T$ of $K$ is described by one of the following two statements: (1) $T$ is a field, algebraic over $k$. (2) $T$ contains an element $x$, transcendental over $k$, and is a ring of quotients of its subring of elements integral over $k[x]$. Further, every integrally closed subring of $K$ is a $Q$-ring.

REMARK. In each of the above two corollaries the first assertion is valid without the assumption of integral closure; a proof will be given in a subsequent paper.

In preparation for a third corollary we must recall some facts about Prüfer rings. It is well known that if $D$ is a valuation ring, then $E$ is a Prüfer ring (e.g., [7, Chapter 1]). It follows that if $D$ is a Prüfer ring, then so is $E$. (If $M$ is a maximal ideal of $E$, then $E_{M}$ is a localization of the Prüfer ring $E_{D-(M \cap D)}$, and is therefore a valuation ring. $E_{D-(M \cap D)}$ is a Prüfer ring because it is the integral closure in $L$ of the valuation ring $D_{M \cap D}$.) It is also well known that if $L$ is a finite extension of $F$, then there are but finitely many prime ideals in $E$ contracting to any given prime in $D$. Hence if $D$ is a Prüfer ring with only finitely many maximal ideals, then the integral closure of $D$ in any finite algebraic extension field of $F$ is a Prüfer ring with only finitely many maximal ideals, a $Q$-domain (Proposition 2). These remarks together with Proposition 3 prove:

COROLlaRY 3. Let $R$ be a Prüfer ring having only finitely many maximal ideals, and let $K$ be an algebraic extension field of the quotient field of $R$. Then every integrally closed subring of $K$ containing $R$ is a ring of quotients of its subring of elements integral over $R$. Further, every integrally closed subring of $K$ containing $R$ is a $Q$-domain.

REMARK. The property that distinguishes $Q$-domains among all Prüfer rings in the Noetherian case (Theorem 2) may be phrased: if $\Delta$ is a set of pairwise co-maximal primes, and the prime $P$ is contained in the set-theoretic union of the members of $\Delta$, then $P$ is contained in some member of $\Delta$. Enticed by the resemblance of this statement to the very familiar one valid for arbitrary commutative rings and arbitrary finite sets $\Delta$, one is tempted to conjecture that this is the property that distinguishes $Q$-domains among Prüfer rings in general. But this conjecture is scuttled by an example already at hand: the ring of all algebraic integers. In this 1 -dimensional $Q$-domain every prime is contained in the set-theoretic union of all the other primes which contract to the same rational integral prime as does the one given. The ring of entire functions of a complex variable affords another counter-example. In this ring every finitely generated ideal is principal; consequently, every pair of elements has a greatest common divisor expressible 
as a "linear combination" of the given elements (as in the integers). With this fact it is an easy exercise to show that every overring is a ring of quotients. However, in this ring every nonunit is contained in a principal maximal ideal; but not every maximal ideal is principal. (For a discussion of the ring of entire functions consult $[4 ; 5]$.)

2. Rings with divisors of zero, a special case. Recall that the total quotient ring of the ring $R$ is $R_{S}$, where $S$ consists of all non-zero-divisors in $R$ and that a ring is integrally closed if it is integrally closed in its total quotient ring. Here an overring of $R$ is a subring of the total quotient ring containing $R$. A ring is a $P$-ring if all of its overrings are integrally closed. A ring of quotients of $R$ is an overring of the form $R_{S}$, where $S$ is a multiplicatively closed set of non-zerodivisors of $R$. (Observe that we say "ring of quotients" for distinction from the general notion of "quotient ring," where the set $S$ is allowed to contain zerodivisors.) A ring is a $Q$-ring if all of its overrings are rings of quotients of the given ring. Notice that the proofs of Lemma 1 and Proposition 1 are valid in the context of rings with divisors of zero provided that we interpret "nonzero element" to mean "non-zero-divisor."' Hence:

Proposition 4. If the integral closure of $R$ is a ring of quotients of $R$, then $R$ is already integrally closed. Consequently, $Q$-rings are $P$-rings.

Some notation: An NZ-ideal is an ideal containing a non-zero-divisor; a maximal 0-ideal is an ideal (necessarily prime) maximal with respect to not containing non-zero-divisors. To replace the localizations used in $\$ 1$ we introduce the following definitions.

Definitions. Let $P$ be a prime NZ-ideal of the ring $R . S(P)$ is the set of non-zero-divisors in the complement of $P$. If $\Delta$ is a set of NZ-primes of $R$, then $R_{\Delta}=\bigcap\left\{R_{S(P)} \mid P \in \Delta\right\}$.

LEMMA 2. For any ring $R, R=R_{\Delta}$, where $\Delta$ consists of the maximal NZideals of $R$.

Proof. Let $x / y$ be an element of the total quotient ring not in $R$. Then $I=\{r \in R \mid r x \in y R\}$ is clearly a proper NZ-ideal of $R$. If $M$ is a maximal ideal containing $I$, then $x / y \notin R_{S(M)}$; for if $x / y=r / s$, then $s \in I$. Hence $x / y$ is not expressible as a fraction with denominator in $S(M)$.

It is quite clear that a "generalized valuation ring" ought to have the property: Of any two non-zero-divisors, one divides the other. With this property alone we are unable to retrieve the properties of valuation rings necessary to recapture the theorems of $\S 1$. For this reason, and also because we are interested mainly in Noetherian rings and their overrings, we make the following definitions.

Definitions. A ring is said to have few zero-divisors if it has only finitely many maximal 0-ideals. A quasi-valuation ring is a ring having few zero-divisors and 
the property: of any two non-zero-divisors one divides the other (or equivalently, for any unit $x$ in the total quotient ring, $x$ or $1 / x$ lies in the given ring).

Remarks. 1. Because of the one-to-one correspondence of those prime ideals of a ring which contain no non-zero-divisors with the prime ideals of the total quotient ring, it is evident that a ring's having few zero-divisors is equivalent to its total quotient ring's having only finitely many maximal ideals. It follows that if a ring has few zero-divisors, then so does each of its overrings. In particular, every overring of a Noetherian ring has few zero-divisors, as does any overring of a finite direct sum of ( $P$ - or $Q$-) domains, the most obvious generalization to rings with divisors of zero of the kinds of rings encountered in $\$ 1$. It is well known that if the ideal $I$ is contained in no member of $\{P\}$, a finite set of primes, then $I$ is not contained in the set-theoretic union of $\{P\}$. Consequently, if $\{I\}$ is a finite set of ideals, no member of which is contained in any member of $\{P\}$, a finite set of prime ideals, then there is an element of $\bigcap\{I\}$ which is in no member of $\{P\}$.(For since no $P$ contains an $I$, no $P$ contains $\bigcap\{I\}$; it follows that $\bigcap\{I\}$ is not contained in the set-theoretic union of $\{P\}$.) We shall use this fact together with "few zero-divisors" in the frequent application of the following three technical lemmas.

Lemma A. Let $M$ and $N$ be prime NZ-ideals of $R$, a ring with few zerodivisors. Then $M$ contains $N$ if, and only if, $R_{S(N)}$ contains $R_{S(M)}$.

Proof. If $M$ contains $N$, then it is clear that $R_{S(N)}$ contains $R_{S(M)}$. Conversely, suppose that $M$ does not contain $N$. Then since $N$ is contained in neither $M$ nor any of the finitely many maximal 0-ideals, there is a non-zero-divisor $s$ in $N-M$. Therefore, $1 / s$ lies in $R_{S(M)}$, but not in $R_{S(N)}$.

LEMMA B. Let $R$ be a ring having few zero-divisors. If $z$ is an element of the total quotient ring of $R$, and $x$ is a non-zero-divisor in $R$, then there is $a u$ in $R$ such that $x+u z$ is a non-zero-divisor. Every NZ-ideal of $R$ has a basis consisting of non-zero-divisors; every finitely generated NZ-ideal of $R$ has a basis consisting of a finite number of non-zero-divisors.

Proof. Let $u$ be an element of $R$ which lies in every maximal ideal of the total quotient ring which does not contain $z$, but lies in no maximal ideal of the total quotient ring which contains $z$. (Here we are making use of the above remarks and the one-to-one correspondence between the maximal ideals of the total quotient ring and the maximal 0-ideals of $R$.) Now it is clear that $x+u z$ can lie in no maximal ideal of the total quotient ring; that is, $x+u z$ is a non-zero-divisor. The first assertion of the lemma is now established. Now suppose that $\left\{z_{i}\right\}$ is a basis for the NZ-ideal $I$. Let $x$ be a non-zero-divisor in $I$. By the first assertion, we may select $u_{i}$ in $R$ such that $z_{i}+x u_{i}$ is a non-zero-divisor. Then $\left\{x, z_{i}+x u_{i}\right\}$ is the required basis for $I$. 
Lemma C. Let $T$ and $W$ be overrings of $R$, a ring with few zero-divisors. Then $T$ contains $W$ if $T$ contains every non-zero-divisor in $W$.

Proof. Let $z$ be an arbitrary element of $W ; z=x / y$, where $x$ and $y$ are in $R$, and $y$ is a non-zero-divisor. By Lemma $\mathrm{B}$, we may select $u$ in $R$ such that $x+u y$ is a non-zero-divisor. Now $z+u=(x+u y) / y$ is a non-zero-divisor in $W$, and therefore lies in $T$. It follows that $z$ lies in $T$.

We are now in a position to establish for quasi-valuation rings all the properties of valuation rings used in $\$ 1$. The proofs (which we record for the sake of completeness) are essentially the same as the proofs of the analogous properties of valuation rings.

Proposition 5. The following statements are valid for the quasi-valuation ring $R$. (1) The prime NZ-ideals of $R$ are linearly ordered by inclusion; $R$ therefore possesses at most one maximal NZ-ideal. (2) Every overring of $R$ is a quasi-valuation ring. (3) If $T$ is an overring of $R$ different from the total quotient ring, and $M$ is the unique maximal NZ-ideal of $T$, then $T=R_{S(M \cap R)}$. (4) $R$ is integrally closed.

Proof. (1) Suppose there is no containment relation between the prime NZideals $I$ and $J$. Since $I$ is contained neither in $J$ nor in any of the finitely many maximal 0 -ideals, we may select a non-zero-divisor $x$ in $I-J$; likewise there is a non-zero-divisor $y$ in $J-I$. Then $x \notin y R$ and $y \notin x R-$ a contradiction of the fact that $R$ is a quasi-valuation ring. (2) Suppose that $T$ is an overring of $R$. If $x$ is a unit in the total quotient ring, then $x$ or $1 / x$ lies in $R$; a fortiori, $x$ or $1 / x$ lies in $T$. So $T$ is a quasi-valuation ring. (3) That $T$ contains $R_{S(M \cap R)}$ is clear. Suppose that $x$ is a non-zero-divisor in $T$. If $x \notin R_{S(M \cap R)}$, then $1 / x \in R_{S(M \cap R)}$ because this ring is a quasi-valuation ring. Further, since $1 / x$ is a nonunit and non-zero-divisor in $R_{S(M \cap R)}, 1 / x \in(M \cap R) R_{S(M \cap R)}$. (Lemma A guarantees that $(M \cap R) R_{S(M \cap R)}$ is the unique maximal NZ-ideal of $\left.R_{S(M \cap R)}.\right)$ Then $1 / x \in M$-impossible, since $x \in T$. Therefore $x \in R_{S(M \cap R)}$. It now follows from Lemma $C$ that $T=R_{S(M \cap R)}$. (4) Let $\bar{R}$ denote the integral closure of $R$. For any $x \in \bar{R}$ we have an equation: $x^{t+1}=r_{0}+r_{1} x+\cdots+r_{t} x^{t}$, with $r_{i} \in R$. Suppose further that $x$ is a non-zero-divisor. If $1 / x \in R$, then division of this equation by $x^{t}$ shows that $x \in R$; if $1 / x \notin R$, then $x \in R$ because $R$ is a quasi-valuation ring. Thus every nonzero-divisor in $\bar{R}$ lies in $R$. It follows that $R=\bar{R}$ (again Lemma C).

DEFINITION. Hereafter we shall sometimes substitute for a proof the statement: a canonical re-reading of the proof of the analogous result in $\$ 1$ yields a proof of $\cdots$. This means that the reader is to substitute "non-zero-divisor" for "nonzero element" and rings of the form $R_{S(P)}$ for the localizations $R_{P}$.

Given the facts of Proposition 5, a canonical re-reading of the proof of Proposition 2 yields a proof of the following proposition. 
Proposition 6. Let $R$ be a ring with few zero-divisors and the property: For every maximal NZ-ideal $M, R_{S(M)}$ is a quasi-valuation ring. Then: (1) Every overring of $R$ has this property. (2) If $T$ is an overring of $R$, then $T$ is of the form $R_{\Delta} ; \Delta$ may be taken to be a set of pairwise co-maximal NZ-primes of $R$. (3) If $R$ has but finitely many maximal ideals, then it is a $Q$-ring.

In preparation for the analog of Theorem 1 we must record a fourth useful technical lemma.

LEMMA D (e.g. [3, Lemma 1]). Let I and $J$ be submodules of an R-module such that for every maximal ideal $M$ of $R, I_{M}$ is contained in $J_{M}$. Then $I$ is contained in $J$. (Here $I_{M}$ denotes the localization of $I$ at $M$, sometimes denoted $I R_{M}$ or $I \otimes_{R} R_{M}$.)

Some technical remarks: Suppose that $K$ is an overring of $R$ and that $S$ is a multiplicatively closed subset of $R-\{0\}$. Then the following points are easily established consequences of the definition of the quotient rings $R_{S}$ and $K_{S}$. (1) $K_{S}$ may be regarded as an overring of $R_{S}$. (2) If $K$ is integral over $R$, then $K_{S}$ is integral over $R_{S}$. (3) If $R$ is integrally closed in $K$, then $R_{S}$ is integrally closed in $K_{S}$. (4) Every overring of $R_{S}$ contained in $K_{S}$ is of the form $T_{S}$, where $T$ is an overring of $R$ contained in $K$.

THeORem 3. Let $R$ be a ring with few zero-divisors. Then $R$ is a P-ring $i$, and only if, for every maximal NZ-ideal $M, R_{S(M)}$ is a quasi-valuation ring.

Proof. If every $R_{S(M)}$ is a quasi-valuation ring, then by Proposition 6, each overring is the intersection of integrally closed rings, and is therefore integrally closed. Now suppose that $R$ is a $P$-ring, and let $K$ denote the total quotient ring of $R$. In the light of the above remarks, for every maximal ideal $M$ of $R, K_{R-M}$ is an overring of $R_{M}$, and every overring of $R_{M}$ contained in $K_{R-M}$ is integrally closed in $K_{R-M}$. If $x$ and $y$ are non-zero-divisors in $R$, then $x / y$ and $y / x$ may be regarded as elements of $K_{R-M}$. Since $x / y$ is integral over $R_{M}\left[x^{2} / y^{2}\right]$, it follows that $x / y$ lies in $R_{M}\left[x^{2} / y^{2}\right]$. We now exploit this fact exactly as in the proof of Theorem 1 to show that $x / y$ or $y / x$ lies in $R_{M}$; there is no need to repeat the argument. We next show that if $M$ is an NZ-ideal, then $R_{S(M)}$ is a quasi-valuation ring. Since $R_{S(M)}$ is a $P$-ring, and $M R_{S(M)}$ is its unique maximal NZ-ideal (Lemma A), there is no loss of generality in assuming that $R$ is a $P$-ring with exactly one maximal NZ-ideal $M$. We have just seen that for any two non-zero-divisors $x$ and $y$ in $R, x \in y R_{M}$ or $y \in x R_{M}$; suppose that $x \in y R_{M}$. For any other maximal ideal $N, x R_{N}=y R_{N}=R_{N}$ since $x$ and $y$ both lie in the complement of $N$. By Lemma $\mathrm{D}, x \in y R$. Thus $R_{S(M)}$ is a quasi-valuation ring.

A canonical re-reading of the proofs of the two corollaries to Theorem 1 now gives analogous corollaries to Theorem 3. The reader perhaps objects to the invocation of Chevalley's theorem (see proof of the first corollary to Theorem 1) 
in the context of rings with divisors of zero. The following argument sets aside this objection. Suppose that $\bar{R}$ is the integral closure of the Noetherian ring $R$, and that there are infinitely many primes in $\bar{R}$ lying over the prime $M$ in $R$. Since there are but finitely many minimal primes in $\bar{R}$ (they are in one-to-one correspondence with those of $R$ ), infinitely many of these primes lying over $M$ contain a single minimal prime $P$. Observe now that $\bar{R} / P$ is an overring of the integral domain $R / P \cap R$ (it is important here that $P$ consist entirely of zero-divisors), that $\bar{R} / P$ is integral over $R / P \cap R$, and that there are infinitely many primes of $\bar{R} / P$ lying over $M / P \cap R$. It follows that there are infinitely many primes in the integral closure of $R / P \cap R$ lying over $M / P \cap R$-a contradiction of Chevalley's theorem. We may now legitimately assert the following two corollaries to Theorem 3.

COROLLARY 1. Let $R$ be a ring having few zero-divisors and such that each overring is of the form $R_{\Delta}^{*}$, where $R^{*}$ is an integral extension of $R$. Then the integral closure of $R$ is a P-ring; if $R$ is Noetherian, then every prime NZ-ideal of $R$ is of rank 1 .

COROLlaRY 2. Let $R$ be a ring having few zero-divisors and in which every NZ-prime has finite rank. Then $R$ is a P-ring if, and only if, every overring of $R$ is of the form $R_{\Delta}$.

As in [3] we shall say that a ring is of restricted dimension 1 if each of its NZ-primes is of rank 1. The remainder of this section is devoted to proving that Noetherian rings of restricted dimension 1 indeed satisfy the hypotheses of Corollary 1 . The reader may therefore assume that all rings considered are overrings of a Noetherian ring of restricted dimension 1 ; it is mainly to simplify the statements of several lemmas that we make the following definition.

Definition. We shall say that the ring $R$ has Property $\mathrm{C}$ if it satisfies the following conditions: (1) $R$ is of restricted dimension 1. (2) $R$ has few zero-divisors. (3) The NZ-ideals of $R$ are finitely generated.

The following theorem is implicit in [2].

COHEN's THEOREM. If a ring has Property $\mathrm{C}$, then so does each of its overrings.

Proof. Let $R$ be the ring in question. Consider first the special case in which $R$ has precisely one NZ-prime. The proof of the "local" version of Cohen's theorem [3, Lemma 3] is given in sufficient generality to handle this special case. Now assume that $R$ is an arbitrary ring with Property $\mathrm{C}$, and let $T$ be a typical overring of $R$. If $M$ is an NZ-prime of $T$, then $R_{S(M \cap R)}$ is a ring of the special case first considered (Lemma A). Since $T_{S(M \cap R)}$ is an overring of $R_{S(M \cap R)}$, $M T_{S(M \cap R)}$ is of rank 1; therefore $M$ is of rank 1 . Consider now an NZ-ideal $I$ of $T$. By the hypothesis on $R$, every prime containing $I \cap R$ is minimal over the ideal. Further, since $R / I \cap R$ is a Noetherian ring, only finitely many primes of 
$R$ can contain $I \cap R$. Denote a typical one of these primes by $P$. Since $T_{S(P)}$ is an overring of $R_{S(P)}$, a ring of the special case, $I T_{S(P)}$ has a finite basis. Let $J$ be the ideal of $T$ generated by a finite set of elements of $I$ which includes a basis of $I \cap R$ and a basis of $I T_{S(P)}$ for each $P$ containing $I \cap R$. For each such $P$, $J T_{R-P}=I T_{R-P}$; for any other maximal ideal $N$ of $R, J T_{R-N}=I T_{R-N}=T_{R-N}$ since $N$ does not contain $I \cap R$. By Lemma $\mathrm{D}, I=J$; that is, $I$ is finitely generated.

Definition. A quasi-valuation ring is discrete if it has exactly one prime $\mathrm{NZ}$-ideal, and that ideal is principal.

Lemma 3. Let $R$ be an integrally closed ring with Property $\mathrm{C}$ and having exactly one NZ-prime. Then $R$ is a discrete quasi-valuation ring.

Proof. We imitate the proof that an integrally closed, 1-dimensional local domain is a discrete valuation ring. Let $M$ and $K$ denote, respectively, the unique NZ-prime and total quotient ring of $R$, and let $M^{-1}=\{z \in K \mid z M \subset R\}$. That $M M^{-1}$ contains $M$ is clear; we claim that this containment is proper. If $M M^{-1}=M$, then since $M$ is finitely generated and contains a non-zero-divisor, by an elementary theorem concerning integral dependence, each element of $M^{-1}$ is integral over $R$; that is $M^{-1} \subset R$. We shall show that this last statement is impossible. Let $x$ be a non-zero-divisor in $M$. Since $M$ is finitely generated and the only prime containing $x$, for some $n, M^{n+1} \subset x R$, and $M^{n} \notin x R$. Select $y \in M^{n}-x R$. It is clear that $y / x \in M^{-1}$ and that $y / x \notin R$. Thus, as claimed, $M M^{-1}$ contains $M$ properly. Now select $z \in M^{-1}$ and $m \in M$ such that $z m \notin M$. We may assume that $z$ and $m$ are non-zero-divisors: if not, replace $z$ and $m$ by $z+u x$ and $m+v x\left(x\right.$ a non-zero-divisor in $M^{2}$ ), the non-zero-divisors prescribed by Lemma B. It is now clear that $z m$ is a unit; without loss of generality, 1 . Then for any $r \in M, r=(r z) m$. Since $r z \in R$, this shows that $m$ generates $M$. We claim that every non-zero-divisor in $M$ is the product of a unit and a power of $m$. If this is not the case, then there is a non-zero-divisor $r \in M$ divisible by every power of $m$. But since for sufficiently large $n, m^{n}$ is divisible by $r$, it follows that $m^{n}$ is divisible by $m^{n+1}$-an absurdity, since $m$ is both a nonunit and a non-zero-divisor. Since every non-zero-divisor in $M$ is of the form claimed, $R$ is clearly a discrete quasivaluation ring.

REMARK. In the light of Theorem 3, Cohen's theorem and the preceding lemma, we may now assert: The integral closure of a Noetherian ring of restricted dimension 1 is a $P$-ring.

LEMMA 4. Let $R$ be a ring with Property $C$ having exactly one NZ-prime ideal. Then the integral closure of $R$ is the intersection of a finite number of discrete quasi-valuation rings.

Proof. Let $\bar{R}$ be the integral closure of $R$. We know that $\bar{R}=\bar{R}_{\Delta}$, where $\Delta$ is the set of NZ-primes of $\bar{R}$, and that for each NZ-prime $P$ of $\bar{R}, \bar{R}_{S(P)}$ is a discrete 
quasi-valuation ring (Lemma 3); we need only show that $\Delta$ is finite. Let $x$ be a non-zero-divisor in the one NZ-prime of $R$. It follows from Cohen's theorem that every NZ-prime of $\bar{R}$ is minimal over the ideal $x \bar{R}$. Since $\bar{R} / x \bar{R}$ is a Noetherian ring (again Cohen's theorem), there are but finitely many such primes.

Lemma 5. Let $R$ be a ring with Property $\mathrm{C}$ and having exactly one NZ-prime. Then if $R$ is integrally closed in a proper overring, $R$ is integrally closed.

Proof. Lemma 5 is the analog for rings with divisors of zero of Krull's Satz 1 [6]. With Lemma 4 at hand Krull's proof applies here with two modifications: (1) Discrete quasi-valuation rings assume the role played by the discrete valuation rings in the integral domain case. (2) Non-zero-divisors are required here at those points of Krull's argument which require nonzero elements.

Theorem 4. Let $R$ be a Noetherian ring of restricted dimension 1 , and let $T$ be an overring of $R$. Then $T=R_{\Delta}^{*}$, where $R^{*}$ is the integral closure of $R$ in $T$, and $\Delta$ is the set of all NZ-primes $P$ of $R^{*}$ such that $R_{S(P)}^{*}$ contains $T$. $\Delta$ includes all those primes $P$ for which $R_{S(P)}^{*}$ is not integrally closed.

Proof. If $R_{S(P)}^{*}$ is not integrally closed, then since $R_{S(P)}^{*}$ is integrally closed in $T_{S(P)}$, it follows that $R_{S(P)}^{*}=T_{S(P)}$ (Lemma 5). In particular, $R_{S(P)}^{*}$ contains $T$, and $P \in \Delta$. Now if $M$ is an NZ-prime of $T, T_{S(M)}$ contains $R_{S\left(M \cap R^{*}\right)}^{*}$. If $R_{S\left(M \cap R^{*}\right)}^{*}$ is not integrally closed, then it contains $T$; that is $M \cap R^{*} \in \Delta$. In this case then, $T_{S(M)}$ contains $R_{\Delta}^{*}$. If $R_{S\left(M \cap R^{*}\right)}^{*}$ is integrally closed, then it is a discrete quasivaluation ring, and so must be equal to $T_{S(M)}$; that is, here too $R_{S\left(M \cap R^{*}\right)}^{*}$ contains $T$, and $M \cap R^{*} \in \Delta$. In either case then, $T_{S(M)}$ contains $R_{\Delta}^{*}$. But since $T=\bigcap\left\{T_{S(M)} \mid M\right.$ an NZ-prime of $\left.T\right\}$, we have that $T$ contains $R_{\Delta}^{*}$. Since $R_{\Delta}^{*}$ contains $T$ by definition, $T=R_{\Delta}^{*}$.

3. Rings with divisors of zero, the Noetherian case. In analogy with the fact that Noetherian $P$-domains are Dedekind rings we shall call Noetherian $P$-rings $D$-rings. A ring of zero-divisors is a ring in which every nonunit is a zero-divisor; that is, a ring which coincides with its total quotient ring. (Recall that if $M$ is a maximal ideal consisting of zero-divisors in the Noetherian ring $R$, then $R_{M}$ is a ring of zero-divisors.) A ring of zero-divisors is of restricted dimension 0 .

THEOREM 5. The following statements are equivalent for the Noetherian ring $R$. (1) $R$ is a D-ring (2) $R$ is integrally closed and of restricted dimension 1 or 0 . (3) For every maximal ideal $M, R_{M}$ is a ring of zero-divisors or a discrete valuation ring. (4) For every maximal ideal $M, R_{M}$ is a D-ring.

Proof. (1) $\rightarrow$ (2) $R$ is integrally closed by hypothesis. If $M$ is a maximal NZideal, then since $M$ has a finite basis of non-zero-divisors (Lemma B), it follows from Theorem 3 that $M R_{S(M)}$ is principal. Therefore $M R_{S(M)}$, and consequently $M$, is of rank 1 ; that is, $R$ is of restricted dimension at most 1. (2) $\rightarrow$ (3) If $M$ 
consists of zero-divisors, then $R_{M}$ is a ring of zero-divisors; if $M$ contains a nonzero-divisor, then $M R_{S(M)}$ is principal (Lemma 3). In the latter case $M R_{M}$ is then principal; that is, $R_{M}$ is a discrete valuation ring. (3) $\rightarrow$ (4) Rings of zerodivisors and discrete valuation rings are trivially $D$-rings. (4) $\rightarrow$ (1) Let $T$ be an overring of $R$, and let $\bar{T}$ be the integral closure of $T$. Now for every maximal ideal $M$ of $R, T_{R-M}$ and $\bar{T}_{R-M}$ are overrings of $R_{M}$, and $\bar{T}_{R-M}$ is integral over $T_{R-M}$ (see remarks preceding Theorem 3); since $R_{M}$ is a $D$-ring, $\bar{T}_{R-M}=T_{R-M}$. It then follows from Lemma $D$ that $T=\bar{T}$; that is, every overring of $R$ is integrally closed.

Remarks. 1. By the second corollary to Theorem 3 , we may add to this list: (5) Every overring of $R$ is of the form $R_{\Delta} .2$. Cohen [2] gives a number of characterizations of Dedekind rings; the substitution of "NZ-ideal" for "nonzero ideal" in many of these yields an analogous characterization of $D$-rings.

THEOREM 6. Thefollowing statements are equivalent for the Noetherian ring $R$. (1) $R$ is a D-ring. (2) Every NZ-ideal is invertible. (3) Every NZ-ideal is a product of primes. (4) Every primary NZ-ideal is a power of its associated prime. (5) The primary ideals belonging to an NZ-prime are linearly ordered by inclusion. (6) If $P$ is an NZ-prime, then there are no ideals properly between $P$ and $P^{2}$.

Proof. It is a routine matter to adapt to the situation at hand the proofs given by Cohen for integral domains. To illustrate we give the proof of the equivalence of (1) and (4) for which we will have use below. Assuming (1), we have that for every maximal NZ-ideal $M, R_{M}$ is a discrete valuation ring. Hence every $M R_{M^{-}}$ primary ideal is a power of $M R_{M}$. It then follows from the one-to-one correspondence between the $M$-primary ideals of $R$ and the $M R_{M}$-primary ideals of $R_{M}$ that every $M$-primary ideal is a power of $M$. Conversely, if every $M$-primary ideal is a power of $M$, then there can be no ideal properly between $M$ and $M^{2}$. It follows that there is no ideal properly between $M R_{M}$ and $\left(M R_{M}\right)^{2}$; or equivalently, that $M R_{M} /\left(M R_{M}\right)^{2}$ is a 1-dimensional vector space over $R_{M} / M R_{M}$. As is well known, this fact implies that $M R_{M}$ is principal; that is $R_{M}$ is a discrete valuation ring. Hence $R$ is a $D$-ring.

Remarks. 1. It is not difficult to see that the integrally closed rings with Property $\mathrm{C}$ encountered in $\$ 2$, and particularly the integral closures of Noetherian rings of restricted dimension 1, enjoy properties (2) through (6) of the above theorem. Recall that if $R$ is a ring of this type, and $M$ is an NZ-prime of $R$, then $M R_{M}$ is principal. With this fact at hand it is an easy matter to establish these properties for $R$; the arguments are substantially the same as those in the case of $R$ 's being Noetherian. 2 . We have already noted that $Q$-rings are $P$-rings (Proposition 4). Given that $D$-rings satisfy (4) of Theorem 6, the equivalence for $D$-rings of the following statements becomes clear: (i) Some power of each NZ-prime is principal. (ii) Each NZ-prime contains a non-zero-divisor which 
lies in no other prime ideal. As is the case for Noetherian domains, it is this property that distinguishes $Q$-rings among $D$-rings. Indeed, a canonical re-reading of the proof of Theorem 2 proves

THEOREM 7. A Noetherian ring is a Q-ring if, and only if, it is a D-ring with the property that some power of each of its NZ-primes is principal.

Suppose that $R$ is the direct sum of finitely many rings $\left\{R_{i}\right\}$, and that $T$ is an overring of $R$. It is a routine matter to verify: (1) $T$ is the direct sum of $\left\{T_{i}\right\}$, where $T_{i}$ is an overring of $R_{i}$. (2) $T$ is integrally closed if, and only if, each $T_{i}$ is integrally closed. (3) $T$ is a ring of quotients of $R$ if, and only if, $T_{i}$ is a ring of quotients of $R_{i}$. (4) $R$ is a $P$ - (Q-) ring if, and only if, each $R_{i}$ is a $P$ - $(Q-)$ ring. This last fact provides us with examples of $P$-and $Q$-rings with divisors of zero: direct sums of rings of zero-divisors and integral domains of the sort studied in $\S 1$. The following example shows that these are not all the $P$-rings.

EXAMPLE. Let $R=Z[x] /\left(2 x, x^{2}\right)$, where $Z$ is the ring of integers and $x$ is an indeterminate. The maximal ideals of $R$ arise from those maximal ideals of $Z[x]$ which contain $x:(p, x)$, where $p$ is a prime integer. Note that $(2, x)$ consists of zerodivisors $\bmod \left(2 x, x^{2}\right)$, and that all zero-divisors in $R$ are in fact contained in this ideal. Since for $p \neq 2, p=2 n+1$, we have that $p x=2 x n+x \equiv x \bmod \left(2 x, x^{2}\right)$; that is, each maximal NZ-ideal is principal. Consequently, $R$ is a $Q$-ring. (One shows this by direct computation or argues as follows: Since each maximal NZ-ideal $M$ is principal, each $R_{M}$ is a discrete valuation ring; hence, by Theorem $5, R$ is a $D$-ring. It then follows from Theorem 7 that $R$ is a $Q$-ring). Observe that since $R$ has but one minimal prime, $x R$, we cannot decompose the ring into a direct sum of the type considered above.

Notice that in the ring of the above example (0) has an imbedded prime component, $(2, x)$. We conclude with the observation that if we rule out rings in which $(0)$ has an imbedded component, then the only $D$-rings are the direct sums considered above.

THEOREM 8. Let $R$ be a Noetherian ring in which (0) has no imbedded component. Then $R$ is a D-ring if, and only if, it is a direct sum of Dedekind rings and rings of zero-divisors; $R$ is a $Q$-ring if, and only if, it is a direct sum of Dedekind rings with torsion class groups and rings of zero-divisors.

Proof. In each case the sufficiency of the direct sum condition is clear; the necessity needs to be proved. Let the primary decomposition of (0) be $Q_{1} \cap \cdots \cap Q_{m} \cap P_{1} \cap \cdots \cap P_{n}$, where the $Q$ 's are primary for maximal ideals, and the $P$ 's are primary for nonmaximal primes. By the hypothesis on (0), each prime belonging to a $Q$ is minimal; it follows that each $Q$ is co-maximal with each $P$ and with each of the other $Q$ 's. Since $R$ is a $D$-ring, for each maximal NZ-ideal $M, R_{M}$ is a discrete valuation ring, and in particular, an integral domain. 
Since the primary decomposition "localizes," we can conclude then that each $P$ is in fact prime, and that no maximal ideal contains more than one $P$. By the Chinese Remainder Theorem, $R$ is the direct sum of the $R / Q_{i}$ and $R / P_{i}$. Each $R / Q_{i}$ is a primary ring. Moreover, since each summand must be a $D$-ring, each $R / P_{i}$ is a Dedekind ring (Theorem 1 ); in the $Q$-ring case each $R / P_{i}$ must be a Dedekind ring with torsion class group (Theorem 2).

Added in proof. R. Gilmer and J. Ohm [Integral domains with quotient overrings, to appear in Math. Ann.] have classified $Q$-domains. Their paper also contains the characterization of Noetherian $Q$-domains given above in Theorem 2 .

\section{REFERENCES}

1. C. Chevalley, La notion d'anneau de décomposition, Nagoya Math. J. 7 (1954), 21-33.

2. I. S. Cohen, Commutative rings with restricted minimum condition, Duke Math. J. 17 (1950), 27-41.

3. E. D. Davis, Overrings of commutative rings. I, Trans. Amer. Math. Soc. 104(1962), 52-61.

4. O. Helmer, Divisibility properties of integral functions, Duke Math. J. 6 (1940), 345-356.

5. M. Henriksen, On the ideal structure of the ring of entire functions, Pacific J. Math. 2 (1952), 178-184.

6. W. Krull, Einbettungsfreie, fast-Noethersche Ringe und ihre Oberringe, Math. Nachr. 21 (1960), 319-338.

7. S. Lang, Introduction to algebraic geometry, Interscience, New York, 1958.

8. O. Zariski and P. Samuel, Commutative algebra, Vols. 1 and 2, Van Nostrand, Princeton, N. J., 1958 and 1960.

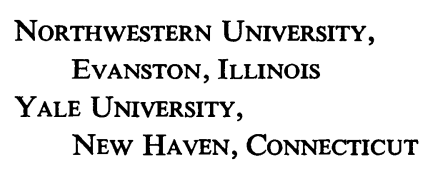

